\title{
Alle origini del vers libre: il caso Marie Krysinska
}

\section{Elena Coppo}

\section{OpenEdition}

\section{Journals}

\section{Edizione digitale}

URL: https://journals.openedition.org/studifrancesi/20596

DOI: 10.4000/studifrancesi.20596

ISSN: 2421-5856

\section{Editore}

Rosenberg \& Sellier

\section{Edizione cartacea}

Data di pubblicazione: 1 décembre 2019

Paginazione: 455-470

ISSN: 0039-2944

\section{Notizia bibliografica digitale}

Elena Coppo, «Alle origini del vers libre: il caso Marie Krysinska», Studi Francesi [Online], 189 (LXIII | III) | 2019, online dal 01 décembre 2020, consultato il 12 novembre 2021. URL: http://

journals.openedition.org/studifrancesi/20596 ; DOI: https://doi.org/10.4000/studifrancesi.20596

\section{(c) (i) ()}

Studi Francesi è distribuita con Licenza Creative Commons Attribuzione - Non commerciale - Non opere derivate 4.0 Internazionale. 


\title{
Alle origini del vers libre: il caso Marie Krysinska
}

\begin{abstract}
The figure of Marie Krysinska, a French poet of Polish origin who lived between the end of the 19th and the beginning of the 20th century, is at the crossroads between the metrical problem of French free verse and the cultural problem of women's place in the history of literature. After an introduction about the relation between women's poetry and free verse in France at the end of the 19th century, this article presents Marie Krysinska and her querelle with Gustave Kahn about the invention of French free verse. The following pages aim to develop a comparison between Kahn and Krysinska from both a theoretic point of view and a poetic one, in light of the most recent studies on the evolution of French poetic forms. While on the one hand their theoretical writings show some important similarities, due to their common symbolist background, on the other hand some formal differences in their poems prove that they followed two different ways of using the free verse and explain the difficulties of understanding Krysinska's poems. The last part of the article resumes the development of the "Krysinska case" in literary studies: violently attacked by critics at the beginning of the 20th century, forgotten by scholars for about fifty years, Krysinska has been rediscovered and reevaluated only in the nineties, and it is now possible to recognize her importance in the literary context of her time, as well as the value of her poetic production.
\end{abstract}

\section{"Femmes-poètes" e "vers libre"}

Dall'inizio del xxI secolo il fenomeno versoliberista è stato oggetto di un crescente interesse in Francia, dove si è assistito al moltiplicarsi degli studi sulla nascita del vers libre, sulle tappe della sua evoluzione e sui teorici e poeti che, tra la fine del XIX e l'inizio del xx secolo, hanno promosso e messo in pratica la liberazione metrica. Al 2008 risale Le Vers libre di Michel Murat, il primo studio sistematico interamente dedicato al verso libero francese; nello stesso anno si è tenuto presso l'Université Paul Verlaine di Metz un convegno dedicato proprio a Le vers libre de 1886 à 1914: naissance, discours et réception, che ha riunito numerosi specialisti e che ha certamente contribuito, insieme all'opera di Murat, a incoraggiare le ricerche in questo ambito. Al tempo stesso, il crescente successo degli studi di genere ha portato a rivolgere l'attenzione anche alle protagoniste femminili del vers-librisme, in passato troppo spesso dimenticate. Proprio in occasione del convegno di Metz, l'intervento di Patricia Izquierdo su Les poétesses de la Belle Époque et le vers libre 1 , pur concentrandosi sul periodo 1900-1914, offre un quadro complessivo della questione.

Gli studi sulla scrittura femminile nella Francia di fine Ottocento e inizio Novecento hanno messo in luce come ai progressi delle donne sul piano politico e sociale e all'aumento del numero delle scrittrici faccia riscontro un atteggiamento di crescente

(1) P. Izquierdo, Les poétesses de la Belle Époque et le vers libre, in Le vers libre dans tous ses états. Histoire et poétique d'une forme (1886-1914), éd. C. Boschian-Campaner, Paris, L'Harmattan, 2009, pp. 109-120. 
misoginia da parte degli intellettuali e degli scrittori contemporanei, che lamentano l'invasione delle cosiddette bas-bleus, e da parte della critica letteraria, che rimane del resto prettamente maschile ${ }^{2}$. Guardando in particolare alle poetesse, Izquierdo osserva che sono due le etichette che vengono loro immancabilmente applicate dalla critica dell'epoca: quella di "imitatrice" e quella di "dilettante". Con la prima viene negata non soltanto l'originalità della poesia composta da donne, ma anche la loro stessa capacità creativa: così, abbondano i giudizi critici che stabiliscono un nesso, per quanto infondato, tra l'opera di una poetessa e quella di un poeta precedente, del quale ella sarebbe "figlia", "allieva" o versione femminile. In secondo luogo, le poetesse sono sempre considerate "in erba", "alle prime armi", indipendentemente dal numero di raccolte pubblicate ${ }^{3}$.

In questo contesto ostile, il desiderio delle poetesse di affermarsi, di essere riconosciute come autrici a pieno titolo, gioca un ruolo importantissimo nelle loro scelte formali, rendendo complesso e spesso contraddittorio il loro rapporto con il neonato verso libero. Era naturale che questo esercitasse su di loro una certa attrazione, in quanto contrappunto formale di quell'emancipazione poetica e personale cui esse indubbiamente miravano; come scrive Marie Dauguet rispondendo all'Enquête internationale sur le vers libre curata da Marinetti: «Le vers libre est en esthétique littéraire le dernier effort de l'évolution individualiste commencée par le romantisme. [...] Il est la forme même du moi intérieur émancipé» ${ }^{4}$. Ma, nonostante questo, la partecipazione femminile al contemporaneo dibattito sulla liberazione metrica è molto rara: già Christine Planté aveva osservato che nel XIX secolo «les femmes écrivains, se conformant apparemment à la modestie de rigueur recommandée à leur sexe, semblent s'être souvent peu souciées de querelles d'école, abstenues de déclarations fracassantes, de proclamations théoriques et de manifestes» ${ }^{5}$, e Izquierdo conferma che «l'écriture des femmes poètes à l'époque est la plupart du temps traditionnelle, avec une métrique et une prosodie régulières malgré quelques libertés parfois», e che a questo si accompagna in genere «un désintérêt affiché pour les questions de versification ${ }^{6}$. Le ragioni di questa difficoltà ad approcciarsi al vers libre vengono individuate in parte nella formazione letteraria femminile - che avveniva per lo più in un contesto familiare ed era decisamente tradizionale, tutta basata sull'imitazione dei classici - ma soprattutto nella «quête d'une légitimité auctoriale» ${ }^{7}$, nella volontà di ottenere una legittimazione, un riconoscimento da parte della critica letteraria contemporanea. A tale scopo era necessario dimostrare di possedere una perfetta padronanza delle forme metrico-prosodiche della tradizione: in questi anni in cui la poesia vive una perenne rivoluzione formale, la critica si dimostra, forse per reazione, fortemente conservatrice; gli scarti rispetto alla norma, già criticati nei poeti, risultano

(2) Cf. in particolare C. Planté, La place des femmes dans l'bistoire littéraire: annexe, ou point de départ d'une relecture critique?, "Revue d'histoire littéraire de la France" 3, 2003, pp. 655-668; C. Planté, M.-E. Thérenty, Masculin/Féminin dans la presse du XIX siècle. Le genre de la critique, in Femmes et critique(s): lettres, arts, cinéma, éd. M. Andrin, L. Brogniez, A. Creusen, A. Favry, V. Gemis, Namur, Presses universitaires de Namur, 2009, pp. 14-28,

(3) Cfr. P. Izquierdo, Les poétesses de la Belle Époque et le vers libre cit., pp. 109-110.

(4) Enquête internationale sur le vers libre et Manifeste du futurisme, dir. F.T. Marinetti, Milano, Edizioni di "Poesia", 1909 , p. 39.

(5) C. Planté, La place des femmes cit., p. 665.

(6) P. Izquierdo, Les poétesses de la Belle Époque et le vers libre cit., pp. 110-111. Infatti, delle 64 raccolte poetiche femminili contate da Izquierdo fra il 1900 e il 1914, ne risultano soltanto 10 interamente composte in forme non tradizionali, e altre 14 solo parzialmente; di tali "forme non tradizionali", poi, solo alcune possono essere effettivamente ricondotte al vers libre, ma la maggior parte sembra piuttosto situarsi nell'ambito del poème en prose (ivi, p. 110).

(7) Ivi, p. 112. 
imperdonabili da parte delle poetesse, e vengono immediatamente interpretati come prove di imperizia o immaturità tecnica ${ }^{8}$.

Tenendo presente tutto questo, si può comprendere meglio l'interesse che suscita la figura di Marie Krysinska, la sola poetessa di questi anni che abbia rivendicato il ruolo di théoricienne del vers libre, nonché la sua invenzione.

\section{Marie Krysinska e la "querelle" con Gustave Kabn}

Della vita di Marie Krysinska non si sa molto. Nata a Varsavia il 22 gennaio 1857, figlia di un eminente avvocato, aveva un grande talento come pianista, e giunse a Parigi da ragazza per proseguire gli studi di musica. Maria Luisa Spaziani, basandosi su una «leggenda orale» riferitale da Paul Fort, racconta che Krysinska, arrivata sedicenne a Parigi, iniziò a comporre poesia per distrarsi nell'attesa del pianoforte che suo padre le aveva comprato, ma che le venne consegnato con un certo ritardo?. Non ci sono dubbi invece sulla sua vivace partecipazione alla vita culturale della capitale: fu membro di numerosi circoli letterari ed artistici (in uno di questi, quello degli Hydropathes, fu notoriamente l'unica donna ammessa), si esibiva regolarmente come pianista al cabaret dello Chat Noir, teneva un salone letterario settimanale nella propria casa, e a partire dal 1881 iniziò a pubblicare i suoi componimenti poetici su alcune riviste letterarie. Nel 1885 sposò il pittore Georges Bellenger e trascorse i primi due anni di matrimonio negli Stati Uniti; tornata in Francia, si dedicò interamente alla letteratura e alla musica, pubblicando raccolte poetiche, romanzi, novelle e saggi. Morì a Parigi il 15 settembre 1908.

Il nome di Marie Krysinska risulta comunque legato innanzitutto alla sua produzione poetica e alla querelle sull'invenzione del verso libero, che la contrappose a Gustave Kahn, tutt'ora considerato l'iniziatore del vers-librisme ${ }^{10}$. Fra il 1881 e il 1883, Krysinska, come si è detto, pubblicò su riviste letterarie parigine - "La Chronique parisienne", "Le Chat noir", "La Vie moderne" - diversi componimenti poetici ${ }^{11}$, caratterizzati da una forma sicuramente non tradizionale, che però l'autrice non si curò, all'epoca, di definire con precisione. Di “verso libero", in quel momento, non si parlava ancora. Fu soltanto nel 1886 che Gustave Kahn pubblicò su "La Vogue", la rivista da lui diretta, i suoi primi testi poetici composti in questa nuova forma, della quale egli si proclamò inventore - costruendo su questo primato tutta la sua carriera letteraria. Quanto a Krysinska, Kahn non le riconobbe alcun ruolo nell'elaborazione della nuova forma poetica: semplicemente la ignorò, rifiutò sempre di pubblicare sulla sua rivista i testi che pure lei gli inviò e, se gli capitò di dover far riferimento a lei, ebbe cura di non chiamarla mai per nome, come per negarle ogni sorta di fama

(8) La critica nei confronti della poesia femminile si somma a quella nei confronti del vers libre nell'ambiguo giudizio formulato da Rachilde - lei stessa, oltretutto, femme de lettres - proprio in merito alla seconda raccolta poetica di Marie Krysinska, Joies errantes: «Le vers libre est un charmant non sens, un bégaiement délicieux et baroque convenant merveilleusement aux femmes poètes dont la paresse instinctive est souvent synonyme de génie» (Rachilde, Joies errantes, par M. Krysinska, "Mercure de France" 56, août 1894, p. 386); a distanza di anni la frase verrà citata, sempre a proposito di Krysinska, anche da Mendès (C. Mendès, Le mouvement poétique français de 1867 à 1900, Genève, Slatkine Reprints, 1993, p. 152).

(9) Cf. M.L. Spaziani, Maria Krysinska inventore del verso libero, in Il simbolismo francese: la poetica, le strutture tematiche, i fondamenti storici, dir. S. Cigada, Carnago, SugarCo, 1992, pp. 379-383: p. 379.

(10) Cf. M. Murat, Le Vers libre, Paris, Champion, 2008, p. 91.

(11) Un elenco completo di questi componimenti viene riportato da S. Whidden, Introduction. Marie Krysinska: Vie libre, vers libre, in M. Krysinska, Rythmes pittoresques, éd. S. Whidden, Exeter, University of Exeter Press, 2003, pp. 1-19: p. 15, n. 52. 
e condannarla all'oblio ${ }^{12}$. Nella nota iniziale alla sua prima raccolta poetica, Rythmes pittoresques, del 1890, Krysinska lamenta proprio questo silenzio, rivendica «l'initiative de ces innovations prosodiques» e la pubblicazione dei «premiers vers libres» nel 1882, e conclude: «Il y eu donc - de la part des confrères manifestants et propagateurs de symbolisme en 1885 - pas mal de perfidie à ne jamais prononcer le nom de Marie Krysinska lorsqu'ils faisaient le dénombrement de leur groupe initial» ${ }^{13}$. Anche nell'Avant-propos della seconda raccolta, Joies errantes, del 1894, Krysinska rivendica «la propriété de l'initiative», attestata da «l'antériorité des dates de publication $(1881-1882) \gg^{14}$.

Ma è nel 1902, con la pubblicazione del saggio di Gustave Kahn Symbolistes et décadents, che il dibattito si fa più acceso. Qui, nel capitolo dedicato alle origini del Simbolismo, ricordando il periodo in cui prestò servizio militare in Tunisia, Kahn racconta di come un giorno gli cadde sotto gli occhi una pagina de «La Vie moderne»: «Je regardais la feuille et $\mathrm{j}$ 'y vis un poème en vers libres, ou typographié tel, poème en prose ou en vers libres, selon le gré, très directement ressemblant à mes essais. Il était signé d'une personne qui me connaissait bien, et voulait bien, moi absent, se conformer étroitement à mon esthétique; je faisais école» ${ }^{15}$.

Pubblicando, l'anno successivo, una nuova raccolta poetica, Intermèdes (1903), Krysinska la fa precedere da un'ampia Introduction nella quale identifica il componimento di cui parla Kahn con il suo Le bibou, pubblicato appunto su "La Vie moderne" il 26 maggio 1883:

«Il était signé d'une personne...» spécifie M. Kahn. C'était moi, «la personne» et ce poême [sic], "Le hibou", fut le seul, en vers libres, que la Vie moderne eût jamais inséré. C'est ainsi que l'on peut rétablir notre signature sous la désignation de une personne qui me connaissait bien, par quoi M. Kahn laisse entendre que son invention, alors strictement inédite, était parvenue à notre connaissance par quelque moyen occulte, sans doute, ou cambrioleur ${ }^{16}$.

Quanto all'affermazione conclusiva di Kahn, «je faisais école», Krysinska risponde divertita: «Heureux climat africain et heureux âge où l'on peut faire école avant d'avoir fait imprimer une seule ligne révélatrice de son esthétique! $\gg^{17}$ : effettivamente, per quanto Kahn potesse aver già elaborato, nel 1883, una sua esthétique, mancavano ancora tre anni alla pubblicazione dei suoi primi vers libres e di un qualche suo intervento teorico in merito. Tuttavia, l'accusa di plagio non fa che riproporre il tradizionale pregiudizio secondo il quale la poetessa non può che essere "imitatrice"; come aveva scritto ironicamente Narcisse Lebeau su "Le Chat noir":

Si Mme Krysinska fut si désinvoltement [sic] éliminée des articles qui battaient la grosse caisse autour des nouvelles préoccupations en art et des noms nouveaux, c'est - uniquement parce qu'elle est une femme (on n'est pas parfait!), mais peut-être aussi parce qu'elle a eu l'indiscrétion d'être première en date dans l'affranchissement du vers; - autrement dit, qu'elle a imité les décadents quatre ans avant que les décadents ne fussent nés pour la littérature ${ }^{18}$.

(12) Cf. ivi, p. 12.

(13) M. Krysinska, Rythmes pittoresques, Paris, Lemerre, 1890, p. VI.

(14) M. Krysinska, Avant-propos, in Ead., Joies errantes, Paris, Lemerre, 1894, pp. V-VIII: p. V.

(15) G. Kahn, Symbolistes et décadents, Paris, Vanier, 1902, p. 29.

(16) M. Krysinska, Introduction sur les évolutions rationnelles, in Ead., Intermèdes, Paris, Messein, 1903, pp. V-XXXIX: pp. XXXIII-XXXIV.

(17) Ivi, p. XXXIV.

(18) N. Lebeau, Rythmes pittoresques, par Mme Marie Krysinska, "Le Chat noir", IX, 457, 18 ottobre 1890, p. 1628. 
Nell'introduzione agli Intermèdes, Krysinska denuncia più volte la sua esclusione, in quanto donna, dal movimento simbolista e versoliberista. I suoi primi componimenti degli anni 1882-1883, scrive, «étaient parfaitement connus de MM. Moréas et Kahn, alors en relation d'amitié avec nous, et qui auraient pu nous mentionner, ne fût-ce qu'à titre de barbare petit Saint-Jean-Baptiste, précurseur» ${ }^{19}$. Invece, il suo nome non era mai stato pronunciato, evidentemente perché «une initiative émanant d'une femme [...] peut être considérée comme ne venant de nulle part, et tombée de droit dans le domaine public» ${ }^{20}$. Tuttavia, questo isolamento forzato viene rivendicato da Krysinska come prova della sua indipendenza rispetto ad un milieu letterario che appare dominato dalla logica delle écoles e dei rapporti fra chefs e disciples. Già nel 1894 Krysinska scrive: «En cette époque où il est fort à la mode d'être chef de quelque école ou, au pis aller [sic], disciple, nous tenons à déclarer notre indépendance littéraire, estimant d'ailleurs qu'un artiste ne vaut que pour la miette de personnalité propre $\gg^{21}$. Nel 1903, poi, la poetessa assume una posizione apertamente critica nei confronti della scuola simbolista e, in particolare, della sua caratteristica obscurité, per la quale i poeti vers-libristes «n'ont aucune excuse à faire valoir, aucune gêne spéciale à invoquer comme en auraient presque le droit les ligotés des prosodies rigoureuses, les suiveurs des maîtres parnassiens, contraints par les exigences conventionnelles $\gg^{22}$. Da parte sua, è fiera che i suoi testi non siano mai stati considerati oscuri o incomprensibili. E conclude: «Sans avoir jamais ambitionné l'emploi de chef d'école, nous déclinons avec énergie le titre de disciple et citons comme témoins de notre indépendance, des dates imprimées qui nous établissent préalable à la formation du groupe novateur, lequel, par l'omission systématique de notre nom, nous a décrétée inexistante et non advenue» ${ }^{23}$.

La durezza di tale querelle, anziché facilitare il confronto fra i suoi due protagonisti, ha finito per congelarli in una netta contrapposizione che non corrisponde necessariamente a realtà. Nei paragrafi successivi si cercherà di fare il punto sulle affinità e sulle differenze fra Gustave Kahn e Marie Krysinska, sia come teorici che come poeti vers-libristes.

\section{Krysinska e Kabn "théoriciens" del vers libre}

Come teorici del verso libero, Kahn e Krysinska appaiono più vicini di quanto si potrebbe pensare: pur dotati di personalità tali da assicurare loro una notevole indipendenza intellettuale, entrambi provengono dal milieu simbolista e ne condividono alcune delle idee fondamentali.

Tra queste c'è innanzitutto il rapporto fra poesia e musica: uno dei pochi punti fermi nella teorizzazione del vers libre, sul quale Krysinska e Kahn sembrano decisamente concordi. Nella Préface sur le vers libre che apre l'edizione del 1897 dei suoi Premiers poèmes, Kahn spiega che per i poeti romantici e parnassiani l'arte più vicina alla poesia era la pittura - una pittura ancora pre-impressionista, plastica, dai

(19) M. Krysinska, Introduction cit., pp. XXXII-XXXIII. Questo passo le valse il soprannome attribuitole ironicamente da Catulle Mendès, quello di «sainte-Jeanne-Baptistine de l'école vers-libriste»: cfr. C. Mendès, Le mouvement poétique français de 1867 à 1900 cit., p. 152.

(20) M. Krysinska, Introduction cit., p. XXI.

(21) M. Krysinska, Avant-propos cit., p. v.

(22) M. Krysinska, Introduction cit., p. Xxvi.

(23) Ivi, pp. XXXVI-XXXVII. 
contorni netti - mentre «la génération suivante fut submergée de musique» ${ }^{24}$, e si dice convinto che la musica abbia avuto una profonda influenza sulla poesia contemporanea e sulla sua in particolare: «Je suis persuadé et sûr, quant à ce qui me regarde, que l'influence de la musique nous amena à la perception d'une forme poétique, à la fois plus fluide et précise, et que les sensations musicales de la jeunesse (non seulement Wagner, mais Beethoven et Schumann) influèrent sur ma conception du vers lorsque je fus capable d'articuler une chanson personnelle» ${ }^{25}$.

Da parte sua Krysinska ebbe, come si è detto, una formazione più musicale che letteraria, e su questo ella insiste molto nei suoi scritti teorici, stabilendo uno stretto legame fra la sua educazione da musicista e la sua poetica, basata sul principio della dissonanza, dell'asimmetria: si definisce infatti come una «spontanée et impulsive musicienne qui essayait de se transposer en poésie» ${ }^{26}$, e spiega: «Musicienne, nous tentions, avec le moyen littéraire de traduire telle impression musicale, avec son caprice rythmique, avec son désordre parfois; usant des ressources prosodiques comme d'ornementations et de parures librement agrafées, sans symétrie obligée» ${ }^{27}$.

Forma d'ispirazione musicale, il vers libre è un verso fatto per essere declamato e ascoltato, piuttosto che scritto e letto; un verso, dunque, pour l'oreille piuttosto che pour l'œil. La preminenza della dimensione musicale e auditiva su quella grafica e visiva emerge chiaramente dagli scritti di Kahn, che afferma che «le poète parle et écrit pour l'oreille et non pour les yeux» e rifiuta, sulla base di questo principio, gli artifici tipografici tradizionalmente legati alla rime pour l' cil $^{8}$. Anche su questo, Kahn e Krysinska si trovano d'accordo: la poetessa si pone l'obiettivo, con la sua poesia, di «atteindre au plus de plaisir pour l'oreille et au plus de musique possible par une eurythmie basée sur le double concours des dispositifs symétriques et des dispositifs asymétriques ${ }^{29}$; inoltre, ella identifica proprio in questa dimensione musicale e auditiva della poesia il suo principale apporto formale: «Notre tentative nouvelle se réduit à un très modeste apport: vers mesurés pour la seul oreille, selon la prononciation moderne usuelle, et assonancés pour l'oreille seulement, avec la faculté de faire rimer les pluriels avec les singuliers et toutes les finales muettes entre elles, quel qu'en soit l'orthographe» ${ }^{30}$. Anche il riferimento, in questo passo, alla pronuncia moderna del francese, e alla necessità che la poesia vi si adegui, trova corrispondenza nella risposta di Kahn all'Enquête di Marinetti, dove egli fa notare che il vers libre «est d'accord avec la phonétique ou avec la prononciation française actuelle à Paris» ${ }^{31}$. Del resto, come scrive ancora Krysinska, «il est normal que la parole parlée ait son reflet fidèle dans la poésie de la même époque», e questo giustifica l'impiego di «une formule moderne, modifiée selon la phonétique actuelle» ${ }^{32}$.

Piuttosto simili appaiono le posizioni teoriche di Kahn e Krysinska anche per quanto riguarda i rapporti del vers libre con i suoi due principali termini di confronto,

(24) G. Kahn, Préface sur le vers libre, in Id., Premiers poèmes, Paris, Mercure de France, 1897, pp. 1-38: p. 8.

(25) Ivi, p. 9.

(26) M. Krysinska, Introduction cit., p. XxxIV.

(27) Ivi, pp. XXII- XXIII.

(28) G. Kahn, Préface cit., p. 31. Va ricordato che nella poesia francese regolare vigeva l'obbligo della rima perfetta sia per l'orecchio che per l'occhio, e quindi il divieto di far rimare insieme parole le cui terminazioni si pronunciavano allo stesso modo ma apparivano graficamente diverse (per esempio, amour e toujours). Così capitava talvolta che i poeti si trovassero a forzare l'ortografia pur di garantire la rima perfetta.

(29) M. Krysinska, Introduction cit., p. XVII.

(30) Ivi, p. XX.

(31) Enquête internationale cit., p. 26.

(32) M. Krysinska, Introduction cit., pp. XIV-XV. 
ossia il verso tradizionale, da una parte, e la prosa dall'altra. Per entrambi, il verso libero va ad affiancarsi al verso tradizionale, non a sostituirsi definitivamente ad esso: entrambi ritengono che la coesistenza delle due forme vada a profitto dei poeti. Essi condividono inoltre l'ambizione, propria della poetica simbolista, di abbattere le tradizionali barriere tra verso e prosa. Nella già citata Préface di Kahn si legge: «Si le vers pseudo-classique ou le vers romantique faible ne se distingue que par la rime, et peut être confondu avec de la prose, le vers libre, plus flottant, pourra être confondu avec de la prose poétique, rythmée et nombrée, avec une sorte de musique. Qu'on en convienne, cela serait déjà mieux, et remplirait davantage notre but» ${ }^{33}$.

Quanto a Krysinska, nell'introduzione a Intermèdes, ella parte dal presupposto che «vers et prose sont tous deux de la littérature avant tout, moyens divers d'un même art, et le mot Poésie, employé pour versification est parfaitement impropre» ${ }^{34}$, dunque la poesia «peut être exprimée dans la plus librement respirante des proses; Bossuet, Chateaubriand, Flaubert sont des poètes» ${ }^{35}$. Può essere interessante notare che anche Kahn cita Flaubert quando, rispondendo all'Enquête promossa da Marinetti nel 1905, afferma che la sua Tentation de Saint Antoine, in prosa, costituisce «le plus beau poème français, une fois ceux d'Hugo comptés, de la fin du XIX siècle» ${ }^{36}$.

L'ambiguità con la prosa è connaturata al vers libre, che rifiuta le categorie tradizionali; tuttavia, proprio la difficoltà di stabilire una distinzione netta rispetto alla prosa ha impedito di cogliere la portata innovativa della poesia di Krysinska, a vantaggio di Kahn.

\section{Krysinska e Kabn poeti "vers-libristes"}

Il primo testo poetico di Krysinska pubblicato in rivista - su "La Chronique parisienne", nel 1881 - è Symphonie des parfums. Così si presenta la sua prima "strofa":

Je veux m'endormir dans le parfum des roses fanées, des sachets vieillis, des encens lointains et oubliés. -

Dans tous les chers et charmeurs parfums d'autrefois. -

Mes souvenirs chanteront sur des rythmes doux, et me berceront sans réveiller les regrets.

Tandis que le morne et spléenétique hiver pleure sur la terre inconsolée,

Et que le vent hurle comme un fou,

Tordant brutalement les membres grêles des ormes et des peupliers,

Je veux m'endormir dans le parfum des roses fanées,

Des sachets vieillis, des encens lointains et oubliés ${ }^{37}$.

Quanto a Kahn, tra le poesie da lui pubblicate su "La Vogue" nel 1886, si può prendere ad esempio l'inizio di Intermède IV:

Timbres oubliés, Timbres morts perdus!

Pas d'une autre glissant à la rue!

Chansons d'amour et vols de grues

Dans d'improbables firmaments!

(33) G. Kahn, Préface cit., pp. 32-33.

(34) M. Krysinska, Introduction cit., p. xxx.

(35) Ivi, p. XXXviII.

(36) Enquête internationale cit., p. 23.

(37) M. Krysinska, Rythmes pittoresques cit., p. 38. 
Les futurs sont à vous, puisque le vent emporte

Vers des cieux, et des lunes, et des flores

Vos petits frissons que nul ne peut clore

Votre âme a glissé sous les lourdes portes

Vers d'imaginaires Lahores ${ }^{38}$.

Nelle poesie di Kahn la novità formale fu subito evidente: mescolanza di versi di misure differenti (dalle 8 alle 12 sillabe, in questo caso) e dalla struttura non convenzionale (come qui i décasyllabes), rimati in maniera irregolare (si veda la rima androgyne dei primi tre versi, e soprattutto il v. 4, irrelato). I primi componimenti di Krysinska, invece, non suscitarono sorpresa da questo punto di vista: vennero infatti percepiti dal pubblico contemporaneo come prosa, ossia come appartenenti al genere, recente ma ormai affermato, del poème en prose. È probabile che la stessa Krysinska inizialmente li considerasse tali, come sembra suggerire la testimonianza dell'amico Georges Rodenbach, che indica il modello formale dei componimenti di Krysinska nelle traduzioni poetiche interlineari:

Or, dans l'esprit de $\mathrm{M}^{\mathrm{me}}$ Marie Krysinska, il ne s'agissait pas du tout de perfectionner ou de révolutionner le Vers; elle-même nous a raconté comment l'idée lui était venue de cette prose rythmée, disposée comme des vers libres. Un jour, en lisant la traduction de Heine par Gérard de Nerval, elle fut fort impressionnée. À chaque vers allemand, dans cette traduction juxtalinéaire, correspondait le sens français qui était, non pas un vers, mais de la prose poétique, puisqu'il traduisait sans césure ni rythme ni rime le vers allemand équivalent.

Or, l'enchaînement de cette prose poétique lui parut donner une apparence de strophes aux membres de phrases inégaux, non sans charme.

C'était quelque chose d'intermédiaire entre la prose et la poésie, ni tout à fait enchaîné, ni tout à fait libre, avec un rythme et une cadence quand même qui en faisait un chant. vers ${ }^{39}$

Alors elle se dit qu'une telle forme lui suffirait pour s'exprimer, sans devoir aller jusqu'au

In realtà, se si prende come modello di poème en prose quello di Baudelaire, si notano alcune significative differenze rispetto ai primi componimenti di Krysinska. In questi ultimi i "paragrafi" sono decisamente più brevi, formati da un unico periodo (come, in Symphonie des parfums, i primi tre), o anche meno (come i successivi), e quindi non sono necessariamente chiusi da un punto fermo ma talvolta da segni di punteggiatura più deboli (virgola, punto e virgola). Queste caratteristiche collocano i primi testi di Krysinska a metà strada fra il poème en prose e il vers libre. Come spiega Michel Murat, Krysinska e Kahn arrivano al vers libre seguendo due percorsi diversi: quello di Krysinska passa attraverso un'ulteriore segmentazione - un découpage - del poème en prose $e^{40}$, mentre Kahn procede per assouplissement del verso regolare, con minor rischio dunque di commistione con la prosa ${ }^{41}$.

Il problema della distinzione fra poème en prose e vers libre riguarda, comunque, soltanto i primi testi di Krysinska, quelli pubblicati in rivista fra il 1881 e il 1883. Krysinska rimase poi lontana dalla scena letteraria francese per un paio d'anni, tra-

(38) G. Kahn, Les Palais nomades, Paris, Tresse et Stock, 1887, p. 54. Proprio in questo testo è stato individuato il point d'émergence della nuova forma: cf. É. Dujardin, Les premiers poètes du vers libre, Paris, Mercure de France, 1922, p. 30, e M. Murat, Le Vers libre cit., p. 104.

(39) G. Rodenbach, La poésie nouvelle. Á propos des décadents et symbolistes, "Revue bleue" 14, t. XLVII, 4 avril 1891, pp. 422-430: pp. 426-427.

(40) M. Murat, Le Vers libre cit., p. 69.

(41) Cf. ivi, p. 105. 
scorsi con il marito negli Stati Uniti; i componimenti da lei pubblicati al suo ritorno, per lo più fra il 1889 e il 1890, e poi confluiti anch'essi nei Rythmes pittoresques, non fanno sorgere alcun dubbio sul loro statuto di vers libres. Se ne riportano qui due esempi molto diversi l'uno dall'altro, tra i più apprezzati e citati dai contemporanei: il primo, Horizons, viene inserito nella sezione Mirages, la prima della raccolta, mentre il secondo, Menuet, fa parte di una sezione dedicata a Les Danses.

\section{Horizons}

Les âpres mâchoires des rochers

Ont dévoré le déclinant soleil

Et la peau aux lourdes rides -

La rude peau des monstres accroupis -

S'éclabousse du sang rose

Que répandit le déclinant soleil.

C'est l'Heure épanouie comme une large Fleur

Où le ciel attristé semble prendre en ses bras

Les monts, les arbres et la mer

Pour d'intimes communions

À l'horizon perdu.

L'olivier pleure aux bords des routes;

Et tout là-bas dans la vallée

Sonnent les gaies couleurs des toitures.

Mais, voici reparaitre la montagne - Reine

Qui porte dans les plis de son long manteau

Les forêts, les vignes et les villes -

Puis, la mer seule

Et dans le beau ciel d'or mourant

Les grandes vagues immobiles

Des Alpes au loin ${ }^{42}$.

Menuet

La soie fleurie

Des longs corsages

Palpite d'amour libertine et discrète.

Les galants paniers

Où éclosent

Des roses

Brodées

Se bercent au rythme lent et mesuré

Du menuet.

Et près de l'oreille: vivante rocaille

Le précieux éventail.

Bat l'aile comme un oiseau

Mourant.

Car le bien aimé, 
(En pourpoint

De satin)

$\mathrm{Y}$ vient roucouler

Un mot si osé.

Vraiment.

Que sous la neige légère des cheveux

Et près des souriantes lèvres

Le gracieux visage devient aussi rose

Qu'une rose

En porcelaine de Sèvres ${ }^{43}$.

I due testi, profondamente diversi non solo nel contenuto, ma anche per tono, forma e stile, dimostrano la versatilità di Krysinska. Il primo è ispirato a un viaggio lungo la Route de la Corniche, la strada costiera che collega Nizza alla Liguria, e ne descrive il maestoso paesaggio in termini altrettanto grandiosi ed eroici; il secondo fa parte invece di un gruppo di componimenti con i quali Krysinska intende riprodurre, in un esercizio di virtuosismo, il ritmo di diversi balli (oltre al Menuet, vi si trovano anche La Pavane, Danse d'Espagne, Danse d'Orient, Javanaises, Danse Slave, La Gigue e Valse).

La forma del primo testo è senza dubbio più vicina, rispetto a quella del secondo, alla prima maniera di Krysinska, ma se ne distingue già in modo significativo: $\mathrm{i}$ versi sono molto più brevi e i periodi coincidono con le strofe anziché con i versi, anche se il découpage rispetta i movimenti della sintassi. Due versi, in chiusura della seconda e della quinta strofa, vengono evidenziati dal rientro tipografico e sembrano dividere la poesia in due sequenze che risultano però asimmetriche per ampiezza e struttura. Mancano completamente le rime, se si esclude quella identica di soleil nella prima strofa, che però si inserisce nella più ampia ripresa del sintagma le déclinant soleil. Questa è comunque l'unica ripetizione: le riprese e iterazioni di sintagmi e di intere frasi erano ben più frequenti nei primi componimenti.

In Menuet la disposizione del testo appare molto più movimentata, con numerosissimi rientri che non corrispondono alla lunghezza dei versi, molti dei quali brevi o brevissimi. Anche il rapporto fra versi e sintassi risulta decisamente più vario, dato che i periodi sono spezzati in segmenti molto brevi, isolando talvolta singole parole; la concordance non si ristabilisce nemmeno a livello delle strofe, visto che l'ultimo periodo si estende dalla seconda alla terza. Un'altra differenza riguarda le rime: come tutti i testi di questa sezione, dall'ispirazione dichiaratamente musicale, Menuet presenta una fittissima rete di rime e quasi-rime interne ed esterne; l'alternanza di versi lunghi e brevi e le rime sono i due principali strumenti di riproduzione del ritmo della danza.

C'è comunque un aspetto che accomuna questi due testi, e che è proprio in effetti dell'intera produzione di Krysinska: la clarté della lingua e delle immagini. I periodi sono costruiti in maniera lineare, il lessico è curato ma nel complesso piuttosto facile, e anche le metafore sono di agevole lettura: è chiara la volontà della poetessa di contrapporsi nettamente all'obscurité dei poeti suoi contemporanei. 


\section{La critica: Marie Krysinska inventrice del "vers libre"?}

Al momento della loro pubblicazione in volume, i Rythmes pittoresques sono accolti con un certo favore: in molti riconoscono ai testi di Krysinska un fascino particolare, fatto di languida grazia e musicalità incantatoria. La loro forma, tuttavia, rimane difficile da definire, anche per coloro che sono più vicini alla poetessa. J.H. Rosny, che firma la Préface alla raccolta, elogia l'opera di Krysinska parlando di «prose rythmée» ${ }^{44}$, ma precisa anche che il volume è il frutto di un lavoro iniziato negli anni 1882-1883, quando l'autrice pubblicò alcuni «morceaux qui offrent la technique des vers libres préconisés en ces derniers temps ${ }^{45}$. E anche Rodenbach, nel suo già citato articolo pubblicato sulla «Revue bleue» nel 1891, prima afferma di aver sentito Krysinska declamare, al circolo degli Hydropathes, «ces premiers vers libres, parus par fragments en 1882, en 1883», e poi racconta, come si è visto, in che modo Krysinska sia arrivata a «cette prose rythmée, disposée comme des vers libres», «forme intermédiaire» 0 «mixte» ${ }^{40}$. Incerti e discordanti sono anche i pareri espressi dalla critica contemporanea, come emerge dai comptes rendus che comparvero sulle riviste dell'epoca e che vengono riportati da Seth Whidden in appendice alla nuova edizione della raccolta da lui curata: $\mathrm{i}$ commenti sono tutti positivi, ma alcuni parlano di «poèmes en prose», «prose rythmée», «prose cadencée», altri di «poèmes en vers et strophes libres», «vers qui n'en sont pas» o «prose scandée» che ha «l'apparence du vers» ${ }^{47}$. Il solo che le riconosca un reale primato formale è Charles Maurras: «Aije dit que Mme Marie Krysinska a tenté le vers libre, il y a huit ans, lorsque nul des poètes d'aujourd'hui n'y songeait encore? Elle a donc inventé presque un art et, tout de suite, elle a su revêtir sa trouvaille d'une grande beauté. Il faut lui en avoir beaucoup de gratitude $\rangle^{48}$.

Prendendo spunto dalla nazionalità polacca di Krysinska, Maurras riflette inoltre sul fatto che la scena letteraria francese è sempre più occupata da scrittori e poeti di origine straniera:

La légion étrangère occupe à main armée tous les postes intéressants des lettres parisiennes. [...] La tradition et l'innovation, l'audace et la sagesse, le quelque chose et le rien sont à Paris pareillement représentés par les Barbares, et le sens symbolique des statues de Shakespeare et de Dante érigées dans nos carrefours, n'est plus à contester. Les Gaulois sont dépossédés de leur langue. Ils l'ont voulu ${ }^{49}$.

Florence Goulesque, alla quale si deve uno dei più ampi e completi studi sulla figura e l'opera di Marie Krysinska, sottolinea che la poetessa si trovò a vivere nel «contexte ambigu d'un cosmopolitisme littéraire doublé d'une méfiance vis-à-vis de l'étranger ${ }^{50}$, e che in particolare il vers libre venne considerato da una buona parte dei letterati contemporanei come il risultato di una «conspiration internationale con-

(44) Cf. M. Krysinska, Rythmes pittoresques cit., pp. VII-VIII: «Votre prose rythmée possède une harmonie délicate; l'euphonie des mots, le système des assonances, la modulation de la période et, d'autre part, la grâce, l'inattendu, la concentration, la saveur des images ne laissent pas un instant de doute sur le caractère nettement et bellement poétique de votre travail».

(45) Ivi, p. VIII, n. 1.

(46) G. Rodenbach, La poésie nouvelle cit., pp. 426-427.

(47) Cf. M. Krysinska, Rythmes pittoresques, éd. S. Whidden cit., pp. 148-156.

(48) C. Maurras, Rythmes pittoresques, "L'Observateur français" 4314, 10 novembre 1890, pp. 1-2 (cfr. M. Krysinska, Rythmes pittoresques, éd. S. Whidden cit., p. 156).

(49) Ivi, pp. 153-154.

(50) F. Goulesque, Une femme poète symboliste. Marie Krysinska, la Calliope du Chat Noir, Paris, Champion, 2001, p. 33. 
tre le vers français» ${ }^{51}$. L'attribuzione delle innovazioni metriche di questi anni all'influenza dei poeti stranieri, che ignorano e dunque corrompono il "genio" linguistico francese, è un luogo comune della critica di questo periodo; fra i poeti innovatori, se ne contano effettivamente diversi di origine straniera, come il greco Jean Moréas, gli americani Vielé-Griffin e Stuart Merrill, i belgi Maeterlinck, Verhaeren e Mockel.

La questione emerge con chiarezza dal rapporto sullo stato della poesia francese che il ministero dell'istruzione commissionò a Catulle Mendès, e che venne pubblicato nel 1903. L'opera riflette, da una parte, l'orientamento del suo autore, poeta parnassiano che già nel 1891, rispondendo all'Enquête di Huret, aveva criticato il primo versoliberismo, derivato a suo parere da un fraintendimento della natura del verso francese causato appunto dall'influenza degli stranieri; dall'altra parte, il rapporto testimonia il carattere conservativo della critica francese d'inizio secolo - per reazione, forse, a una rivoluzione delle forme poetiche che durava ormai da molto tempo e sembrava spingersi sempre oltre. Ebbene, trattando la questione dell'origine del vers libre, «un incident $\gg^{52}$, nel percorso della poesia francese, Mendès si sofferma prima sulla figura di Della Rocca de Vergalo, luogotenente peruviano in esilio a Parigi, al quale attribuisce un'importanza decisamente eccessiva e senza riscontro altrove, poi su quella di Marie Krysinska che, scrive, «fera bien de ne point prétendre à la gloire d'avoir été une novatrice $>^{53}$. Come ha notato Ida Merello, non è un caso che Mendès citi due stranieri all'origine del vers libre: «il suggère quand même qu'il s'agit d'une technique relâchée, que les étrangers créent à cause de leur faiblesse dans la langue française $\gg^{54}$.

In questo contesto Marie Krysinska, polacca trapiantata in Francia, si vede attribuire dunque il doppio pregiudizio riservato alle donne e agli stranieri, lo stesso che si può ricavare anche dal saggio Le Symbolisme di André Barre - in origine una tesi di dottorato - del 1911, dove si legge: «Les essais de Marie Krysinska sont des fantaisies à l'origine sans prétention [...]. Ils manifestent avant tout l'indolence féminine en matière d'art, l'antipathie de la femme pour tout travail fini. Ils sont l'expression instinctive et nullement théorique d'une double nonchalance, celle d'une femme et celle d'une Slave» ${ }^{55}$.

Se l'opera di Mendès ben rappresenta la componente della critica letteraria ostile al vers libre, coloro che invece ne sono fautori attribuiscono invariabilmente la sua invenzione a Gustave Kahn, eventualmente anticipato da Rimbaud e Laforgue, ma certo non da Krysinska. La question Krysinska rimane comunque aperta, fino all'intervento di Édouard Dujardin, che nel suo saggio Les premiers poètes du vers libre, del 1922, si propone di fare chiarezza. Dujardin si dimostra molto sicuro, sia nell'individuare in Marine e Mouvement di Rimbaud i primi esempi di vers libres mai pubblicati, sebbene riconosciuti come tali solo a posteriori ${ }^{56}$, sia nel dichiarare che $i$

(51) Ivi, p. 158

(52) C. Mendès, Le mouvement poétique cit., p. 150.

(53) Ivi, p. 152.

(54) I. Merello, Pour une définition du vers libre, in Le vers libre dans tous ses états cit., pp. 123-132: p. 130.

(55) A. Barre, Le Symbolisme. Essai historique sur le mowvement symboliste en France de 1885 à 1900 , Paris, Jouve et Cie, 1911, p. 335. Patricia Izquierdo nota che il vers libre viene spesso considerato, per la sua origine straniera, una forma ibrida e contro natura, «un peu comme la femme écrivain, souvent cosmopolite» (P. Izquierdo, Les poétesses de la Belle Époque et le vers libre cit., p. 117). Donna e straniera, Krysinska viene talvolta indicata anche come ebrea, anche se non è certo che lo fosse; Laurent Tailhade in particolare scrisse pagine velenose su «la juive polonaise Marie Krysinska» (cfr. M. Krysinska, Rythmes pittoresques, éd. S. Whidden cit., p. 9, n. 31). Al pregiudizio contro gli stranieri si affianca quindi l'antisemitismo che, com'è noto, è molto diffuso nella Francia dell'epoca. Anche Kahn, pur essendo a tutti gli effetti francese, viene spesso considerato straniero in quanto di origini ebraiche (cfr. l'elenco di poeti stranieri stilato da Charles Maurras e riportato ivi, p. 154).

(56) Cf. É. Dujardin, Les premiers poètes du vers libre cit., p. 29: «Saluons! Ce sont les premiers vers libres qui aient jamais été publiés». 
componimenti di Krysinska sono poèmes en prose scambiati a torto per vers libres. L'autore ammette che il modo in cui Kahn ha affrontato la questione non ha fatto che dare maggior credito alla poetessa: infatti, accusandola di plagio, egli ha implicitamente riconosciuto che i testi da lei composti e pubblicati in rivista nei primi anni Ottanta costituivano vers libres, e tanto basterebbe per attribuirle la precedenza, almeno stando alle date di pubblicazione. Ma non è così: «De l'examen de ces poèmes il n'y a qu'une conclusion à tirer. Ce n'est pas du vers libre, c'est du poème en prose» ${ }^{57}$. Tuttavia, i criteri da lui forniti per distinguere queste due forme non risultano granché convincenti ${ }^{58}$.

D'altro canto, Dujardin osserva che «la bonne foi de Marie Krysinska ne semble d'ailleurs pas incontestable» ${ }^{59}$ : alcuni dei suoi testi pubblicati in rivista, al momento del loro inserimento fra i Rythmes pittoresques, sono stati infatti modificati dalla poetessa, che ha ridotto la misura dei "versi" andando a capo più spesso. Dujardin illustra il procedimento nel caso di Symphonie en gris, la cui prima pubblicazione risale al 1882 su «Le Chat noir». L'incipit si presentava allora così:

Plus d'ardentes lueurs sur le ciel alourdi, qui semble tristement rêver.

Les arbres, sans mouvement, mettent dans le loin une dentelle grise.

Sur le ciel qui semble tristement rêver, plus d'ardentes lueurs.

Al momento della pubblicazione tra i Rythmes pittoresques, lo stesso testo assume invece un aspetto diverso:

Plus d'ardentes lueurs sur le ciel alourdi,

Qui semble tristement rêver.

Les arbres, sans mouvement,

Mettent dans le loin une dentelle grise.

Sur le ciel qui semble tristement rêver,

Plus d'ardentes lueurs.

In relazione al terzo "verso" - «Les arbres, sans mouvement» - Dujardin si domanda: «Est-il possible d'imaginer que ces quatre mots constituent un vers? $\gg^{60}$, senza peraltro spiegare perché ciò sia impossibile. Egli definisce comunque il découpage una «petite tricherie», e conclude ironicamente: «Il ne suffit pas, Madame, pour faire des vers libres, de passer à la ligne à chaque membre de phrase» ${ }^{61}$.

(57) Ivi, p. 19

(58) Cf. ivi, p. 17: «Le poème en prose diffère du vers, d'abord en ce que ses pieds rythmiques sont généralement d'un rythme moins prononcé, ensuite et surtout en ce qu'ils ne sont pas ordonnées dans l'unité resserrée du vers».

(59) Ivi, p. 20.

(60) Ivi, p. 20, n. 1.

(61) Ivi, p. 21. La questione è stata accuratamente illustrata da Seth Whidden, che ha osservato come nel gesto di Krysinska si possa vedere anche, semplicemente, «une expérimentation poétique» (M. Krysinska, Rythmes pittoresques, éd. S. Whidden cit., pp. 14-17). Di fatto, considerata la mancanza di una distinzione netta fra poème en prose e vers libre, «si on admet que l'opération de Krysinska est une "tricherie", il serait quand même difficile d'expliquer envers quoi il s'agit d'une tricherie» (S. Whidden, Sur la supercherie de Marie Krysinska. Vers une lecture sérieuse de "Symphonie en gris", in Le vers libre dans tous ses états cit., pp. 79-88, p. 85). Anche Ida Merello ha notato l'inconsistenza dell'argomentazione di Dujardin, che «nel momento in cui dovrebbe fornire indicazioni oggettive di distinzione tra verso e prosa, [...] si richiama in realtà ad un'ineffabile immediatezza prosastica», finendo così per dimostrare «come sia del tutto impossibile stabilire regole di codificazione del moderno verso libero e rigide distinzioni tra prosa e poesia» (I. Merello, La nascita del verso libero, "Studi francesi” 138, anno XLVI, III, settembre-dicembre 2002, pp. 570-587: p. 586). 
Stabilendo l'illegittimità delle rivendicazioni della poetessa, Dujardin ha effettivamente chiuso per molto tempo la question Krysinska, che nei decenni seguenti non è stata ripresa che molto raramente, per lo più senza mettere in discussione la sua argomentazione. Questa è stata ripresa in particolare da Suzanne Bernard nel suo studio del 1959 sul poème en prose: l'autrice, non senza manifestare un certo disprezzo per la poetessa polacca, ripropone la questione del découpage avvenuto tra la prima e la seconda versione di Symphonie en gris, affermando che nell'effettuare la segmentazione Krysinska «paraît oublier complètement que le vers libre, dans son principe, refuse l'enjambement [...] et doit constituer une unité»: ecco perché «Les arbres, sans mouvement» non può essere considerato un verso, perché non costituisce un'unità semantica completa. Secondo Bernard «c'est évidemment une prétention bizarre, et qui prouve un manque inquiétant d'oreille et de goût, que de s'imaginer "faire" des vers libres en coupant ainsi chaque phrase en deux»: «en somme nous avons là une véritable démonstration (par l'absurde) prouvant qu'on ne fabrique pas du vers libre en débitant en tranches, au petit bonheur, une phrase de prose» ${ }^{62}$.

Bernard fa riferimento alla concezione del verso propria dei primi vers-libristes, basata sulla concordance fra verso e sintassi (che trova riscontro nella teoria di Kahn: il verso come «fragment le plus court possible figurant un arrêt de voix et un arrêt de sens» $\left.{ }^{63}\right)$; in quest'ottica, il fatto che l'accapo separi il soggetto della frase, les arbres, dal suo verbo, mettent, appare inaccettabile. Tuttavia non c'è ragione di giudicare i testi di Krysinska sulla base dei criteri formali stabiliti dal suo rivale. La concordance verso-sintassi (ossia la coincidenza verso-pensiero) rappresentò per i primi poètes novateurs un ottimo strumento di legittimazione del vers libre, in grado di provarne la razionalità, contro l'accusa di arbitrarietà rivoltagli dai suoi detrattori. Krysinska, da parte sua, non sembra avvertire tale necessità; in questo senso è interessante una sua dichiarazione, tratta da un suo articolo del 1900 e citata anche da Whidden: «Quant à la disposition graphique, la division linéaire n'étant plus motivée par la rime ou l'assonance, qui sont facultatives, elle est arbitraire, en effet. Mais, là encore, c'est l'arbitraire commun à tous les moyens d'art» ${ }^{64}$. In un periodo in cui i poeti fautori del vers libre si sforzano di giustificare la nuova forma, affermando che essa è dotata di regole più ferree di quelle della metrica tradizionale e che presenta persino, rispetto ad essa, un maggior grado di difficoltà, la posizione di Krysinska appare decisamente moderna.

Dopo Bernard, la querelle Kahn-Krysinska viene ripresa da J.C. Ireson, che ne espone i vari passaggi nella sua tesi del 1962 dedicata a Kahn. Ireson classifica i primi testi di Krysinska come poèmes en prose, affermando che a distinguerli dal «vers libre authentique» non è la mancanza di unità o di concordance, bensì «leur rythme moins prononcé» e «l'absence de la rime». Quanto alla produzione successiva di Krysinska, si tratta per lui di vers syllabiques, ossia di versi tradizionali liberamente mescolati. Egli ammette che Kahn «a eu sans doute moralement tort de dénigrer les tentatives de sa rivale», ma non le riconosce alcun ruolo nell'elaborazione della nuova forma ${ }^{65}$.

(62) S. Bernard, Le poème en prose de Baudelaire jusqu'à nos jours, Paris, Nizet, 1959, pp. 372-373. Che invece sia possibile arrivare al vers libre per semplice découpage di un testo in prosa, lo ha dimostrato Apollinaire con la sua Maisons des morts, prima pubblicata come racconto in prosa e poi, nel 1909, in versi: un atto al quale Murat attribuisce un valore fondante nella storia del vers libre (cfr. M. Murat, Le Vers libre cit., p. 117).

(63) G. Kahn, Préface cit., p. 26.

(64) M. Krysinska, L'Évolution poétique. Devant l'Académie, "Revue universelle" 5, 2 février 1901, pp. 102 103: p. 103 .

(65) J. C. Ireson, L'ouvre poétique de Gustave Kabn (1859-1936), Paris, Nizet, 1862, pp. 86-89. 
Ireson osserva che il nome di questa poetessa «paraît aujourd'hui bien effacé»: la critica l'ha ormai sostanzialmente dimenticata, in Francia e ancora di più all'estero. In Italia, in particolare, il valore della poesia di Krysinska non ha avuto alcuna possibilità di essere riconosciuto, a causa innanzitutto della politica di "Poesia", la rivista fondata nel 1905 e diretta da Filippo Tommaso Marinetti; organo ufficiale del movimento futurista, essa ebbe un ruolo fondamentale nell'importazione del vers libre in Italia, tant'è vero che proprio da "Poesia" partì, nel 1905, l'Enquête internationale sur le vers libre. Kahn, al quale Marinetti guardava come a un maestro, fu un assiduo collaboratore della rivista ${ }^{66}$, e il suo nome vi compare costantemente: gli sono dedicati interi articoli, vengono pubblicati numerosi suoi testi e, nel momento in cui la rivista lancia l'inchiesta sul verso libero, la domanda rivolta ai letterati italiani è la seguente: «Quali sono le vostre idee pro e contro il così detto "verso libero" in Italia, derivato dal "vers libre" francese che Gustave Kahn ha creato in Francia?». La consacrazione di Kahn a inventore del vers libre non potrebbe essere più completa. Quanto a Marie Krysinska, il suo nome non viene citato che un'unica volta sulle pagine di "Poesia", proprio nell'ambito dell'inchiesta, quando Gian Pietro Lucini la nomina, en passant e fra parentesi, nel rivendicare l'originalità della propria produzione poetica: «Quando, infatti, nel 1888 uscivano i "Semiritmi" di Luigi Capuana, a cui ben volentieri accordo la priorità, io aveva già composto, in parte, ciò che in quel tempo chiamava "Armonie sinfoniche", ignorando il nome di "Semiritmi" e di "Rythmes pittoresques" (Maria Krysinska) $\gg^{67}$.

Tuttavia, nella riscoperta e nella rivalutazione di Marie Krysinska, una parte di merito sembra dover essere attribuita a un'italiana, Maria Luisa Spaziani. È stata lei, nei primi anni Novanta, a riaprire la questione della priorità, prima dedicando alla poetessa polacca una delle interviste immaginarie che compongono il suo volume Donne in poesia ${ }^{68}$, del 1992, poi rivendicando apertamente, in un saggio pubblicato in quello stesso anno, il suo «ruolo di primissima teorica e creatrice del verso libero» ${ }^{69}$. Spaziani presenta la "questione Krysinska" come «un importante caso letterario che si tinge dei colori dell'errore giudiziario storico» ${ }^{70}$, denuncia l'oblio nel quale è caduta la sua produzione poetica e critica, misconosciuta sia in Polonia che in Francia, per non parlare dell'Italia, e il fatto che il suo nome compaia al massimo in una nota nei capitoli sul verso libero, mentre Kahn ne viene presentato come l'incontestato inventore. A dire la verità l'autrice, nell'indicare in Krysinska l'unica vera inventrice del vers libre - attribuendo una portata rivoluzionaria al poemetto Le Hibou, del 1883, e dichiarando che «il verso libero di Maria Krysinska non è assolutamente da confondere con il poemetto lirico o il versetto in prosa o biblico ${ }^{71}$ - dimostra più entusiasmo che accuratezza, ma in questo modo riporta all'attenzione una figura che ne ha avuta certamente meno di quanto meritasse.

Negli anni successivi, un nuovo interesse per la figura e la poesia di Marie Krysinska si è registrato negli Stati Uniti, grazie in particolare a due studiosi, Florence Goulesque e il già più volte citato Seth Whidden, che le hanno dedicato le loro

(66) Sui rapporti di Kahn con Marinetti e con "Poesia", cfr. L. Nissim, Gustave Kabn et la revue "Poesia" de Filippo Tommaso Marinetti, in Gustave Kabn (1859-1936), dir. S. Basch, Paris, Garnier, 2009, pp. 459-482.

(67) Enquête internationale cit., p. 113. Va comunque ricordato che Lucini è stato anche autore di un'opera monumentale come Ragion poetica e programma del verso libero (1908) e che nelle circa 700 pagine di questo volume Krysinska non viene mai citata.

(68) Cf. M.L. Spaziani, Donne in poesia. Interviste immaginarie, Venezia, Marsilio, 1992, pp. 67-79.

(69) M.L. Spaziani, Maria Krysinska cit., p. 379.

(70) Ibidem.

(71) Ivi, p. 381. 
tesi di dottorato rispettivamente nel 1997 e nel 2000, e hanno continuato ad occuparsene anche in seguito ${ }^{72}$. Nessuno dei due è arrivato alla conclusione che Krysinska sia stata la sola vera inventrice del vers libre: Goulesque preferisce attribuirle una forma poetica propria, intermedia fra vers libre e poème en prose $e^{73}$; quanto a Whidden, egli definisce Krysinska come «une des femmes-poètes les plus importantes du dix-neuvième siècle», «une des premières à écrire de la poésie en vers libre» e «la seule femme parmi les novateurs de la versification française de la deuxième moitié du dix-neuvième siècle» ${ }^{74}$.

Nel frattempo la figura di Marie Krysinska è stata naturalmente rivalutata anche in Francia, come testimoniato dal convegno internazionale che le è stato dedicato nel 2008, presso la Bibliothèque Polonaise di Parigi ${ }^{75}$; sebbene la riscoperta della sua poesia sia avvenuta inizialmente nell'ambito degli studi di letteratura di genere, la critica metricologica francese non ha potuto fare a meno di prenderne atto, con la conseguenza che oggi chiunque voglia tracciare una storia della nascita del vers libre in Francia deve necessariamente dedicare a Marie Krysinska qualcosa di più di una nota a piè di pagina. La tanto dibattuta questione della priorità passa ormai in secondo piano, mentre rimane una figura di donna e di scrittrice decisamente eccezionale, che partecipò attivamente all'avanguardia letteraria simbolista mantenendo però una posizione indipendente e molto moderna; inoltre, fu una delle poche poetesse di Francia a prendere parte al processo di liberazione e di rinnovamento delle forme poetiche, e l'unica a rivendicare lo statuto di théoricienne. Oltretutto, al di là del dibattito formale, si può cogliere ancora oggi la bellezza della sua poesia, dotata di un fascino pittoresque e al contempo di una lingua chiara, priva di obscurité.

ELENA COPPO
Università degli Studi di Padova

(72) Cf. F. Goulesque, Une femme poète symboliste cit.; Seth Whidden ha invece curato l'edizione dei Rythmes pittoresques del 2003 e, più di recente, la raccolta dei Poèmes choisis suivis d'Études critiques, Saint-Étienne, Publications de l'Université de Saint-Étienne, 2013.

(73) «'ai préféré présenter le pourquoi et le comment de la démarche de Krysinska, sa recherche d'une forme bien à elle, entre vers libre et poème en prose, plutôt que de démontrer une supériorité artificielle» (F. Goulesque, Une femme poète symboliste cit., p. 195).

(74) S. Whidden, Introduction cit., p. 1.

(75) Gli atti del convegno sono stati riuniti in Marie Krysinska. Innovations poétiques et combats littéraires, dir. A. M. Paliyenko, G. Schultz, S. Whidden, Saint-Étienne, Publications de l'Université de SaintÉtienne, 2010. 